\title{
EL PERIODISMO MÉDICO EN LA PERIFERIA ESPAÑOLA DURANTE EL PRIMER TERCIO DEL SIGLO XX: APROXIMACIÓN A LA BIOGRAFÍA Y OBRA DE JOSÉ SÁNCHEZ POZUELOS (MURCIA, C. 1885 - 1936)
}

\author{
José López González \\ Servicio Murciano de Salud \\ jlg025@hotmail.com \\ José Miguel Sáez Gómez \\ Universidad de Murcia \\ jmsaez@um.es
}

Recibido: 6 mayo 2014; Aceptado: 2 septiembre 2014.

Cómo citar este artículo/Citation: López González, José y José Miguel Sáez Gómez (2015), "El periodismo médico en la periferia española durante el primer tercio del siglo XX: Aproximación a la biografía y obra de José Sánchez Pozuelos (Murcia, c. 1885 - 1936)", Asclepio 67 (1): p085. doi: http://dx.doi.org/10.3989/asclepio.2015.11

RESUMEN: Se estudia la trayectoria biográfica y la contribución de José Sánchez Pozuelos (Murcia, c. 1885-1936) al periodismo médico español del primer tercio del siglo XX, una época que en Murcia, como en el resto de España, fue un período de resurgir cultural y científico manifestado, entre otras cosas, por la edición de numerosas revistas médicas que pretendieron difundir entre los profesionales la producción científica más relevante, nacional y extranjera. En ese marco el médico José Sánchez Pozuelos, perteneciente a la burguesía murciana que detentaba el poder político local, e identificado con ideologías de carácter conservador y religioso, interesado al mismo tiempo por sacar del atraso a su tierra, fundó y dirigió Murcia Médica (1915-1918) y Estudios Médicos (1920, 1924-34), revistas que se convirtieron en la herramienta de difusión de la actividad de la Real Academia de Medicina de Murcia, pero también de otros profesionales españoles, y de los trabajos más relevantes procedentes de diversas publicaciones nacionales y extranjeras; Estudios Médicos llegó a ser además la revista médica nacional de mayor tirada. El ambiente de inestabilidad social que precedió a la sublevación militar de 1936 y el enfrentamiento bélico que le siguió truncaron el desarrollo de estas publicaciones y de los profesionales que las impulsaron.

PALABRAS CLAVE: Historia de la Medicina; Siglo XX; Periodismo médico; Sánchez Pozuelos, José (c. 1885-1936); Murcia (España); Murcia Médica (1915-1918); Estudios Médicos (1920, 1924-1934).

Copyright: () 2015 CSIC. Este es un artículo de acceso abierto distribuido bajo los términos de la licencia Creative Commons Attribution-Non Commercial (by-nc) Spain 3.0. 


\title{
THE MEDICAL JOURNALISM IN THE SPANISH PERIPHERY DURING THE FIRST THIRD OF THE TWEN- TIETH CENTURY: AN APPROACH TO THE BIOGRAPHY AND WORKS OF JOSÉ SÁNCHEZ POZUELOS \\ (MURCIA, C. 1885-1936)
}

\begin{abstract}
The biographical career of the physician José Sánchez Pozuelos and his contribution to the medical journalism in the first third of the twentieth century have been studied. In Murcia, as in the rest of Spain, this was a period of cultural and scientific excellence manifested, among other things, by the publication of many medical journals with the aim of disseminating the most relevant scientific production, the national as well as the foreign one. In this context, doctor José Sánchez Pozuelos who was belonging to the bourgeoisie that held the political local power and was also identified with conservative and religious ideologies as well as concerned with overcoming the backwardness of his homeland, founded and directed Murcia Médica (1915-1918) and Estudios Médicos (1920, 1924-1934). Both journals became the disseminating tool for the activity of the Murcia's Royal Academy of Medicine, but also for other Spanish professionals and for the most relevant papers from various national and international publications; Estudios Médicos also came to be the Spanish medical journal with the largest circulation. The atmosphere of social unrest that preceded the military uprising of 1936 and the war that followed it truncated the development of these publications and the careers of the professionals that promoted them.
\end{abstract}

KEY WORDS: History of Medicine; 20th century; Medical journalism; Sánchez Pozuelos, José (c. 1885-1936); Murcia (Spain); Murcia Médica (1915-1918); Estudios Médicos (1920, 1924-1934).

\section{INTRODUCCIÓN}

José Sánchez Pozuelos (c. 1885 - 1936), hasta ahora desconocido, es un personaje clave para reconstruir la historia del periodismo médico y para comprender las estrategias de comunicación y de divulgación del conocimiento por parte de las instituciones médicas durante el primer tercio del siglo XX. Sánchez Pozuelos fue fundador, secretario de redacción, redactor y director de Murcia Médica (1915-18), el primer órgano oficial de la Real Academia de Medicina de Murcia, y también fundó y dirigió el segundo órgano de la institución, Estudios Médicos (1920, 1924-34). En este trabajo estudiamos la biografía de José Sánchez Pozuelos y la estructura y contenidos de Murcia Médica con el objetivo de comprender los esfuerzos realizados en comunidades periféricas, carentes de instituciones académicas o científicas relevantes, por incorporarse al ritmo general de España y Europa. La revista Estudios Médicos ha sido objeto de estudio con anterioridad (López y Sáez, 2005; Pérez Gómez, 2011), por lo que solo nos referiremos a ella brevemente. Partimos de la hipótesis de que ante la ausencia de instituciones que den cauce a la actividad científica, cobran relevancia las iniciativas individuales entendidas, claro está, en el marco histórico y social concreto en que se desarrollan (Olagüe, 2005; Carrillo, 2013). Así cabe interpretar la contribución, en colaboración con otros profesionales, de José Sánchez Pozuelos.

Nacido hacia 1885 ejerció profesionalmente en Murcia, conoció el reinado de Alfonso XIII, la Dictadu- ra de Primo de Rivera (1923-1931) y murió al final de la Segunda República (1931-1936), en el mismo momento de la rebelión de parte del ejército contra la legalidad republicana. Las circunstancias de ese periodo histórico marcaron la biografía de nuestro protagonista. Para lograr nuestros objetivos se ha procedido al análisis de las revistas en las que participó Sánchez Pozuelos y se han utilizado como fuentes de información, además, la prensa diaria y los fondos documentales de los archivos Municipal de Murcia y General de la Región de Murcia.

La aspiración de Murcia de contar con una Facultad de Medicina para formar a sus profesionales y para que sirviera de motor científico, es antigua. De ella hay constancia documental desde $1622^{1}$. Sin embargo esta aspiración no se vería colmada hasta más de trescientos años después (Marset y Sáez, 2013). El papel de las asociaciones y academias como impulsoras de la actividad científica en sus orígenes ha sido ya estudiado; por citar solo algunos ejemplos, véanse los trabajos de Rumeu de Armas (1980) o de Capel, Sánchez y Moncada (1988). En Murcia, desde 1759 cuando apareció la primera Academia de Medicina, hasta 1969 cuando se puso en marcha la Facultad de Medicina, las sucesivas Academias intentaron ocupar ese espacio que la ausencia de la Facultad dejaba libre (Sáez y López, 2005; Sáez, Marset y Pérez, 2013; Sáez y Marset, 2013). Estas Academias, como también hicieron el resto de las europeas utilizaron las publicaciones periódicas como medio de comunicación entre 
los profesionales y de difusión del conocimiento médico y de sus propias actividades (López Piñero, 1991).

La obra de Sánchez Pozuelos se inscribe en una trayectoria que se inició en el último cuarto del siglo XVIII, cuando aparecieron las publicaciones médicas seriadas en Murcia. Las primeras fueron las Memorias editadas por la Academia Médica Murciense de San Raphael, existente en la capital desde 1759 y al menos hasta 1772 (Sáez Gómez, 1992). En cuanto a publicaciones propiamente periódicas, la primera revista médica aparecida en la Región de Murcia fue La Unión de las Ciencias Médicas que ejerció como órgano de la Academia Médico-Farmacéutica de Cartagena desde enero de 1881 (Ferrándiz Araujo, 1994), casi ciento cincuenta años después de la primera publicación de estas características en España. La Real Academia de Medicina y Cirugía de Murcia, sin embargo, no contó con un órgano de expresión hasta la iniciativa de Sánchez Pozuelos y otros profesionales a partir de una fecha tan tardía como 1915. Sus iniciativas coincidieron con la inserción de la incipiente dinámica científica regional en los avatares de la ciencia española, ciencia que alcanzó sus cotas máximas en la Segunda República.

Una manifestación del dinamismo de todo este período fue el periodismo médico que se desarrolló en Murcia. Entre 1881, momento en que apareció la primera publicación periódica de carácter científico sanitario unos años antes del nacimiento de Sánchez Pozuelos, y 1936, fecha en que murió nuestro protagonista, el periodismo profesional sanitario tuvo en Murcia unos años de auge, con la aparición de un total de 28 revistas, entre las que tuvieron un papel destacado las impulsadas por él (López, Sáez y Valera, 2002).

\section{APROXIMACIÓN A LA BIOGRAFÍA DE JOSÉ SÁNCHEZ POZUELOS}

Todavía no tenemos constancia de la fecha de nacimiento de José Sánchez Pozuelos. Debió de ser hacia 1885, ya que se licenció en Medicina en Madrid en 1909 con la calificación de sobresaliente ${ }^{2}$. La Universidad de Madrid, con las de Valencia y Granada fueron los centros donde se formaron los estudiantes murcianos hasta la creación de la Universidad de Murcia en 1915. Padre de un niño $0^{3}$ y una niña ${ }^{4}$ en su dimensión privada, su personalidad pública se abrió a diversas actividades de carácter religioso, político (en parte ligada a la anterior), y también lógicamente, a una actividad profesional, científica e institucional como médico.

En lo que a sus actividades religiosas se refiere, le conocemos en 1913 como miembro de la directiva de la asociación católica «Patronato de San José Obrero», junto a otras personalidades, entre ellas el también médico Francisco Giner Hernández ${ }^{5}$. Fue además Vocal de la Comisión de la Congregación Mariana del Patronato del Carmen en 19206. En 1929 fundó la Asociación de Caballeros Carmelitanos ${ }^{7}$ de la que fue Presidente hasta su muerte en 1936; como tal en 1932 dirigió El Barrio del Carmen, periódico mensual editado por la mencionada Asociación ${ }^{8}$. Por último en este aspecto, entre $1930^{9}$ y 1936 fue Mayordomo de la Cofradía de la Sangre y su ViceSecretario en $1935^{10}$.

Sánchez Pozuelos se identificó ideológicamente con un partido conservador portavoz de la burguesía, bajo la dirección de Juan de la Cierva Peñafiel, alcalde de Murcia en 1895 y varias veces ministro ${ }^{11}$, y su hijo Juan de la Cierva Codorníu ${ }^{12}$. La unidad de la burguesía murciana continuó durante toda la década de 1920-1930 en torno al "ciervismo» y al partido de Unión Patriótica que sostuvo la Dictadura y favoreció depuraciones políticas sobre algunos cargos y corporaciones poco afines (Rodríguez Llopis, 1999, pp. 430-7). Sánchez Pozuelos formó parte de la Juventud Conservadora de Murcia, de la que en 1913 y 1914 fue Vocal $^{13}$, y en 1915 Vicepresidente ${ }^{14}$ y Director de Patria, el órgano de la Organización ${ }^{15}$. Fue su Secretario en 1916 y 1917. Posteriormente ingresó como concejal en el Ayuntamiento de Murcia (ver figura 1) donde entre 1928 y 1930 tuvo responsabilidades de Alcalde interino ${ }^{16}$, Segundo Teniente de Alcalde $^{17}$, Presidente de las Comisiones de Beneficencia y Sanidad, y de Hacienda. En sus funciones de concejal actuó como interventor delegado en las Oficinas Sanitarias contra la Anquilostomiasis de las pedanías murcianas de Puebla de Soto, desde que fue inaugurada en mayo de 1928 (Guillamón, 1929, p. 16), y Rincón de Seca, inaugurada en enero de $1930^{18}$. En 1929 dirigió las obras de la nueva Casa de Socorro de Murcia en el Paseo del Malecón ${ }^{19}$. En 1930 redactó el reglamento de Beneficencia y Sanidad que aprobó la Dirección General de Sanidad ${ }^{20}$. En 1935 fue nombrado miembro de la Junta Provincial de Beneficencia ${ }^{21}$. Dentro de esta actividad política y social, en diciembre de 1928 aprovechó su revista Estudios Médicos para proponer al Ayuntamiento de Murcia la concesión de la Medalla de oro de la Ciudad ${ }^{22}$ a Juan de la Cierva Codorníu, José Pérez Mateos ${ }^{23}$, y Mariano Ruiz Funes García ${ }^{24}$.

Tras la muerte de Jaime Ferrán en Barcelona, Sánchez Pozuelos propuso, en la Sesión de 27 de noviembre de 1929 de la Comisión Permanente del Ayuntamiento de Murcia, que tanto una plaza de la ciudad como el Dispensario Antituberculoso Municipal llevaran su nombre ${ }^{25}$. En 1930 inició una suscripción popular para costear un Homenaje al ingeniero Cierva Codorníu con la colocación de un busto en el Jardín de Floridablanca y una lápida de bronce, instalada en 1935 , que dio su nombre a la conoci- 
Figura 1. José Sánchez Pozuelos, teniente de alcalde (a la izquierda) despachando en el Ayuntamiento de Murcia con el alcalde, Marqués de Ordoño. Levante Agrario, 8 septiembre 1929

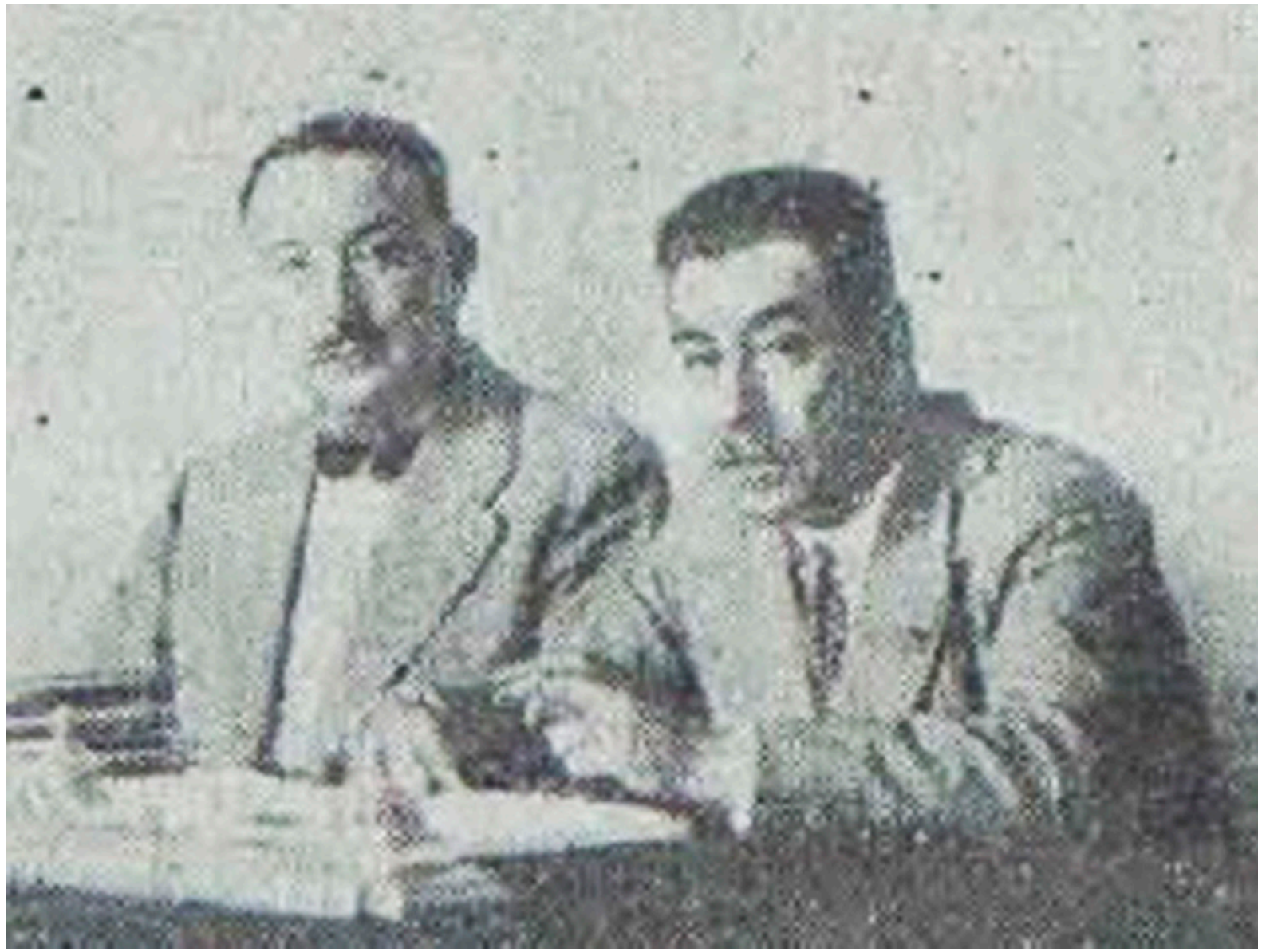

da como Plaza del Rollo en la ciudad de Murcia26. Terminó militando en Acción Popular Murciana, un partido de la derecha confesional católica integrado en la coalición CEDA -Confederación Española de Derechas Autónomas-(Moreno Fernández, 1996, pp. 459-69). Sánchez Pozuelos formó parte de su dirección al menos desde 1933 (Moreno Fernández, 1987), y al iniciarse la Guerra Civil apoyó al ejército sublevado, por lo que fue juzgado ${ }^{27}$, condenado a muerte por rebelión militar ${ }^{28}$ y ejecutado el 16 de diciembre de $1936^{29}$.

De la actividad profesional de Sánchez Pozuelos existe escasa información. En junio de 1915 sustituyó a José Riquelme Paredes, médico Titular de Beniel, mientras estuvo en Granada por asuntos profesionales ${ }^{30}$. Como la mayor parte de los profesionales del momento ejerció la medicina privada en $\operatorname{Murcia}^{31}$ y, al menos en 1920, obtuvo una financiación económica -250 pesetas- del Ayuntamiento de Murcia en consig- nación por vacuna ${ }^{32}$. Desde 1922 era propietario de una Farmacia y Laboratorio (ver figura 2) que regentaba su pariente Julio Sánchez Pozuelos ${ }^{33}$.

Tampoco hay excesiva información sobre su actividad institucional. En 1915 ingresó como Académico Correspondiente en la Real de Medicina y Cirugía de Murcia con el discurso "Algunas consideraciones sobre la fisiología del corazón». Poco después ingresó también como Corresponsal en las Academias de Granada $(1917)^{34}$ y de Higiene de Cataluña $(1925)^{35}$. Participó así mismo en la Sección Científica del Colegio Provincial de Médicos, de la que fue Vocal en 1920 y Secretario en 1921.

Muchos profesionales murcianos del período que nos ocupa, tras finalizar sus estudios de medicina adquirieron una sólida formación científica gracias al apoyo institucional que les proveyó de becas de estudio en España o en el extranjero. Véanse por ejemplo los casos del cardiólogo Luis Calandre (Sebastián 
Figura 2. Publicidad de la farmacia Sánchez Pozuelos, ilustración de Luis Gil de Vicario. Murcia Deportiva, 1 abril 1923 [extraordinario], p. 40

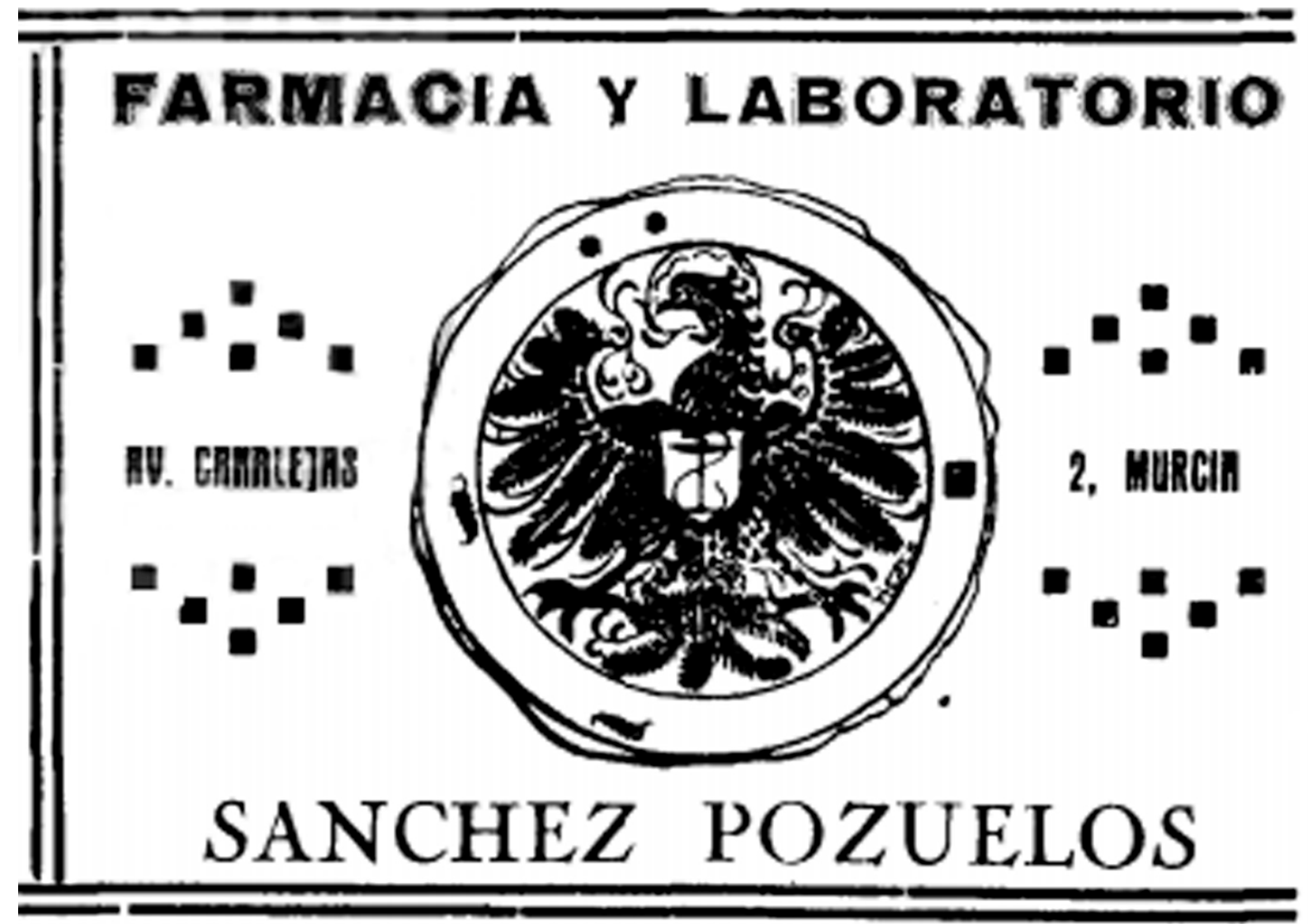

Raz, 2011), el histólogo Antonio Pedro Rodríguez Pérez (Rodríguez Ruiz, 2012), los psiquiatras Román Alberca y Luis Valenciano (Marset, 2008), los epidemiólogos Laureano Abaladejo García y Mariano Abril Cánovas, los traumatólogos Juan González-Aguilar y Manuel Clavel Nolla, el oftalmólogo Antonio Ros o el farmacólogo y cardiólogo Rafael Méndez, entre otros $^{36}$. Esa formación les permitió acceder a la Universidad o a instituciones públicas sanitarias y desde ellas desarrollar una amplia actividad profesional y científica. Otros como Martínez Ladrón de Guevara (López González, 2012) o quien nos ocupa, José Sánchez Pozuelos, sin esa formación, al finalizar sus estudios se reintegraron a su tierra natal donde se dedicaron al ejercicio privado de la profesión, sin por ello renunciar a intervenir directamente en la evolución de la medicina y la sanidad, participando en las instituciones sanitarias (Sáez y López, 2005; Sáez, Marset y Pérez, 2013), impulsando publicaciones profesionales y científicas (López y Sáez, 2005), con- tribuyendo a los inicios de la formación universitaria (Valera, 2005) o implicándose en la gestión política.

La producción científica de Sánchez Pozuelos fue escasa, pues la mayor parte de sus publicaciones están relacionadas con su actividad social religiosa (p.e. el Reglamento de la Asociación de Caballeros Carmelitanos, impreso en Murcia en 1931$)^{37}$ o con su actividad como responsable de revistas médicas, aspecto este que analizaremos en una sección aparte. Sus publicaciones estrictamente científicas solo fueron tres, todas ellas fechadas entre 1915 y 1916, en la revista Murcia Médica (ver figura 3), y relacionadas con la especialidad de Aparato Circulatorio en la que ejerció: a la primera (Sánchez Pozuelos,1915a) sobre arritmias, siguió otra para describir el tratamiento de un caso clínico de estrechez mitral (Sánchez Pozuelos, 1915b), y finalizó con la publicación de lo que fue su discurso de ingreso en la Academia de Medicina de Murcia (Sánchez Pozuelos, 1916). 
Figura 3. Caricatura de José Sánchez Pozuelos realizada por Gil de Vicario. Murcia Médica, 1917, 3 (25)

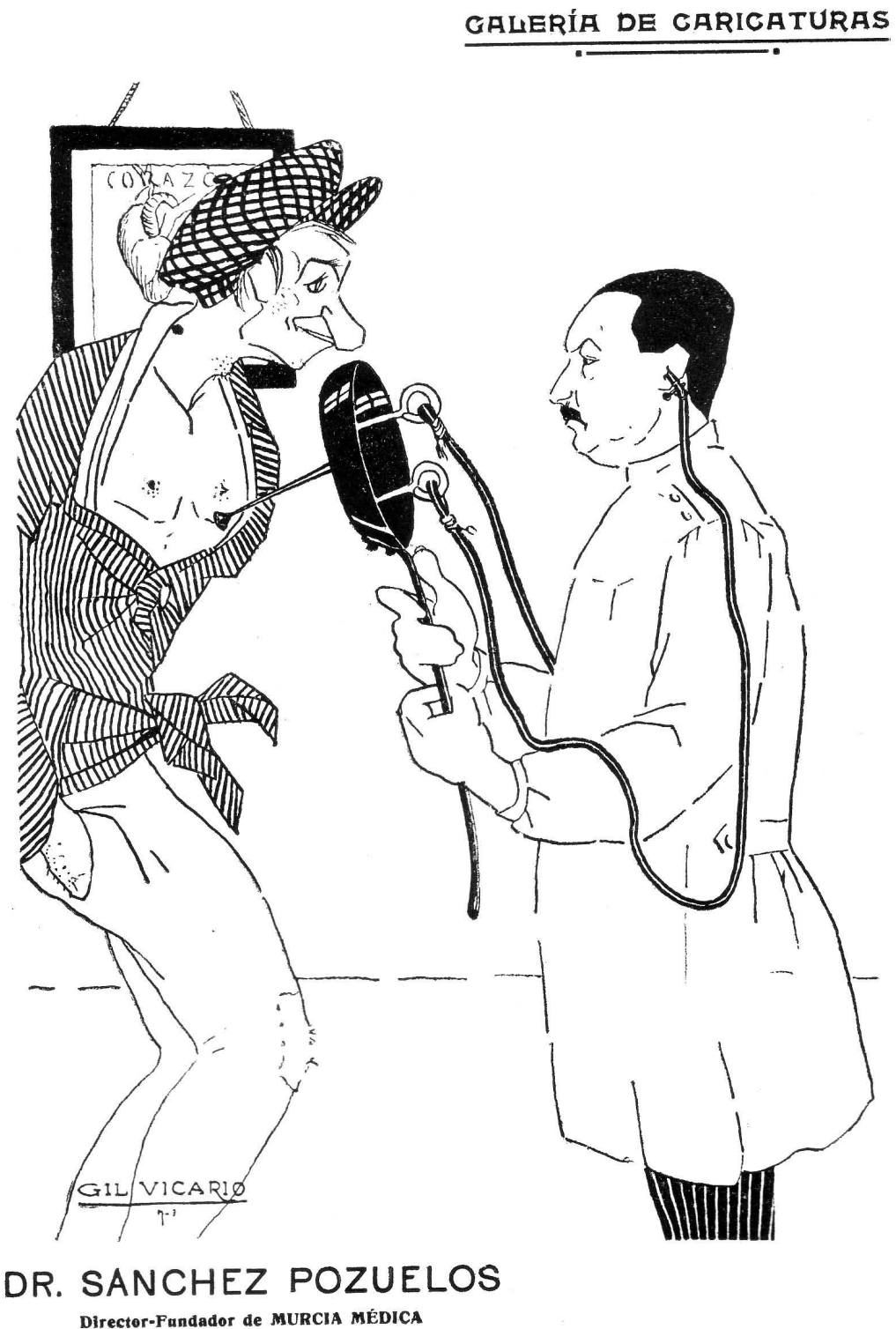

\section{JOSÉ SÁNCHEZ POZUELOS, PUBLICISTA}

Si Sánchez Pozuelos no tuvo una amplia producción, en cambio no cabe duda de que la comunicación científica fue su auténtica vocación. No se debe perder de vista que la obra de Sánchez Pozuelos se enmarca en la conocida como «edad de plata de la cultura española» y que, como ya señalaron López Piñero y Terrada (1991), coincide con el momento culminante del periodismo médico como medio de comunicación científica y profesional. Consciente Sánchez Pozuelos de las posibilidades que ofrecían las publicaciones periódicas, en los primeros años de su actividad profesional se interesó por las publicaciones médicas, lo que le llevó a fundar y dirigir dos de ellas, Murcia Médica (1915-18) y Estudios Médicos (1920, 1924-34), que se convirtieron como ya se anunció en los primeros órganos oficiales de expresión de la Real Academia de Medicina y Cirugía de Murcia. La iniciativa de Sánchez Pozuelos no es única en el panorama español, pero tampoco lo habitual. Se ha demostrado que en 
este momento no es la iniciativa particular la que impulsa la mayor parte de las publicaciones científicas, sino instituciones y asociaciones varias entre las que, además, solo excepcionalmente se cuentan las Academias de Medicina (López Piñero y Terrada, 1991).

Murcia Médica, fundada en abril de 1915, fue una revista mensual que se definía como de «medicina, cirugía y especialidades». En ella Sánchez Pozuelos figura como secretario de redacción y después como director. Junto a él otros dos médicos: Antonio Guillamón Conesa como director y Juan Antonio Martínez Ladrón de Guevara en la administración. Tras cuatro años de publicación desapareció en 1918. En su declaración de principios afirma no perseguir fin lucrativo alguno y sí fomentar y estimular el estudio de la medicina ${ }^{38}$. Para tal fin publicó artículos científicos seleccionados, bien originales bien de otras fuentes, tanto españolas como de otros países, además de informar de acontecimientos científicos y noticias de interés general. También creó un premio al mejor artículo científico original, como hicieron en la misma época otras publicaciones similares.

Con el fin de dar una amplia difusión a su revista, los fundadores decidieron establecer unos precios de suscripción (5 pts. anuales) muy bajos, lo que suscitó una polémica en su tercer año de vida cuando otras dos revistas, que no ha sido posible identificar, insinuaron su relación con la industria farmacéutica. Los directores de la publicación murciana reaccionaron con un artículo que detallaba cómo se administraba Murcia Médica, y exponía cómo habían conseguido gestionar una revista médica mensual con un precio de venta al público inferior a su coste material ${ }^{39}$.

A lo largo de sus cuatro años de vida la revista fue modificando tanto su aspecto como sus contenidos. Así, en enero de 1916 dejó de diferenciar las funciones de sus promotores, que en adelante figuraron los tres como "directores fundadores», y se identificaron además como "Académicos corresponsales de la Real de Medicina y Cirugía de Murcia». Por otra parte adoptó una nueva imagen que sería la definitiva y aumentó de cuarenta a sesenta las páginas de texto por número. También renovó sus contenidos y comenzó a reproducir artículos completos de otras revistas y no solo reseñas como venía haciendo. El segundo cambio apareció en mayo de 1917 (ver figura 4).

A partir de ese momento, bajo el título de la revista aparecerá como subtítulo la frase, en mayúsculas, "ÓRGANO OFICIAL DE LA REAL ACADEMIA DE MEDICINA Y CIRUGÍA DE MURCIA». A este aspecto dedicaremos un apartado especial en el presente trabajo.

Fue Murcia Médica la primera de las revistas murcianas en presentar una redacción constituida por un grupo de médicos encargados cada uno de una espe- cialidad. Para coordinar este grupo de profesionales José Sánchez Pozuelos asumió las funciones de secretario de redacción. Desde el comienzo la redacción estuvo formada por catorce médicos para trece especialidades diferentes. Con el tiempo se incorporaron otras tres especialidades (dermatología, enfermedades de la nutrición y urología) y desapareció una (sifiliografía), con sus respectivos responsables (Palazón, Amorós y Egea, y Conejero respectivamente). Para la especialidad de medicina general llegaron a sucederse hasta tres responsables (Rey Larramendi, Cano y Vinader). El resto de especialidades (aparato circulatorio, aparato digestivo, aparato respiratorio, biología, cirugía general, electrología, ginecología, oftalmología, otorrinolaringología y pediatría) permanecieron con los mismos redactores desde el principio.

Otros dos aspectos son destacables en Murcia Médica: sus colaboradores y la iconografía. La colaboración de Murcia Médica se incrementó desde veintinueve individuos al comienzo hasta cincuenta y dos que figuran en el último número, tras cuatro años de vida. La relación de colaboradores aparece en la primera página, a continuación de la redacción, con indicación de sus apellidos seguidos de la localidad donde ejercían. En enero de 1916 se introdujo otro apartado en esta parte de la revista, la colaboración americana que hasta el último número estuvo compuesta por los mismos tres médicos de Buenos Aires. En septiembre de 1917 se incorporó el último colaborador a Murcia Médica; a partir de entonces y hasta noviembre de 1918, último fascículo que ha sido posible estudiar, figuran cincuenta y dos colaboradores, cuarenta y nueve españoles y tres americanos. La distribución geográfica la lidera Madrid que aportó un $44 \%$, le sigue Murcia con un 17\%, Valencia con un $11 \%$, Salamanca con casi un $8 \%$, Buenos Aires con casi un $6 \%$, y el resto con menos del $4 \%$ cada una.

En lo que a iconografía se refiere, cada número cuenta con páginas publicitarias, láminas con retratos de los colaboradores de Murcia Médica, y con láminas que ilustran los artículos. Además la revista incluye entre el texto también caricaturas.

En Murcia Médica se publicaron 44 páginas con retratos de 46 autores (ver tabla 1). Las fotografías de los colaboradores están al principio de cada fascículo. Son fotograbados de buena calidad, la mayoría de veces recortados y adheridos con cola a una hoja de papel normal de la revista. Tan solo en tres ocasiones se imprimieron directamente en papel satinado de mayor calidad. Se publicaron mensualmente sin interrupción y coincidieron con el autor del primer artículo de ese mismo número ${ }^{40}$. Esta manera de dar comienzo al fascículo con el retrato del autor del artículo que abría ese número se da en otras revistas de la época en todo el territorio español, sirvan los ejemplos de $L a$ Clínica Moderna y de Clínica y Laboratorio de Zaragoza (Gastón Barcos y Ubieto Artur, 1996, 99). 
Figura 4. Primera página de Murcia Médica 1917, 3 (26)

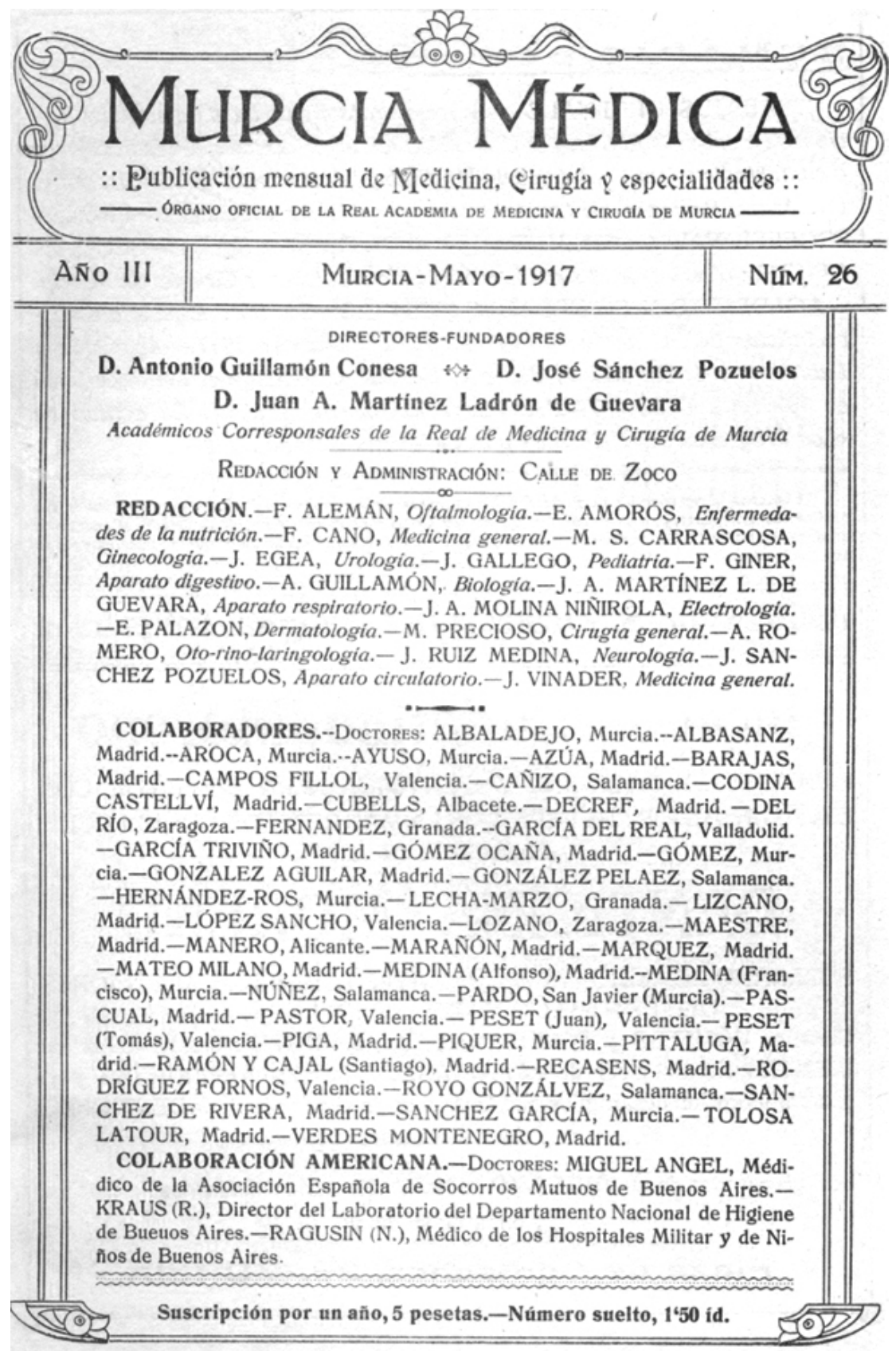

Al igual que los fotograbados, las caricaturas aparecen con anterioridad en otras revistas médicas de todo el territorio nacional. En Murcia Médica se publicaron caricaturas en todos los números desde el 23 hasta el último estudiado (noviembre de 1918, número 44), a excepción de los 27,33 y 39 . La serie se inició con los tres fundadores a los que siguieron los redactores y algunos colaboradores (ver tabla 2). Dieciocho de estos dibujos son originales de Luis Gil de Vicario (Sáez, Marset y López, 2008) y existe uno de dife- rente estilo y firma, la caricatura de Cano Soria en el número 31, cuyo autor no ha podido ser identificado.

Murcia Médica dejó de publicarse en 1918, por motivos desconocidos y aparentemente escindiéndose en tres nuevas revistas: Revista de Tisiología y Especialidades (1919-1926) -dirigida por Juan Antonio Martínez Ladrón de Guevara-, Estudios Médicos (1920, 1924-1934) -que dirigió José Sánchez Pozuelos-, y La Conferencia Médica (1921-1922) -bajo la di- 
Tabla 1. Relación alfabética de retratos en Murcia Médica

\begin{tabular}{|c|c|c|c|}
\hline FOTOGRABADO DE & № & FOTOGRABADO DE & № \\
\hline Albaladejo, L (Murcia) & 18 & Marañón Posadillo, G (Madrid) & 35 \\
\hline Albasanz Echevarría, S (Madrid) & 3 & Marín Agramunt, J (Valencia) & 40 \\
\hline Ángel Cremades, M (Buenos Aires) & 26 & Márquez, M (Madrid) & 5 \\
\hline Ayuso Andreu, F (Murcia) & 38 & Medina, $\mathrm{A}$ & 8 \\
\hline Barajas, L (Madrid) & 15 & Medina Romero, $\mathrm{F}$ & 9 \\
\hline Bové Piqué, E (Barcelona) & 32,41 & Muñoz Urra, F (Talavera de la Reina) & 33,39 \\
\hline Del Cañizo, A (Salamanca) & 21 & Navarro Blasco, A (Madrid) & 36 \\
\hline Fernández Martínez, F (Granada) & 16 & Núñez García, A (Salamanca) & 14 \\
\hline García del Real (Valladolid) & 23 & Pastor Reig, R (Valencia) & 19 \\
\hline García Triviño, F (Madrid) & 22 & Peset, J (Sevilla) & 11 \\
\hline Gómez Ocaña (Madrid) & 12 & Peset, T (Valencia) & 11 \\
\hline González Aguilar, J (Madrid) & 44 & Piga Pascual, A (Madrid) & 31 \\
\hline González Peláez, J (Salamanca) & 27 & Piquer Hernando, S (Murcia) & 37 \\
\hline Gresa de Camps, S & 42 & Príncipe de Asturias & 27 \\
\hline Gresa de Mirambell, A (Barcelona) & 42 & Recasens, S (Madrid) & 10 \\
\hline Hernández-Ros, C (Murcia) & 28 & Rodríguez Arías, B (Barcelona) & 30 \\
\hline Irigoyen Arruti, T.A. (San Sebastián) & 43 & Royo Gonzálvez, M (Salamanca) & 4 \\
\hline Lecha-Marzo, A (Granada) & 17 & Sainz Iriondo, R (San Fernando) & 25 \\
\hline López Albo, W (Bilbao) & 24 & Sánchez García, E (Murcia) & 29 \\
\hline López Sancho, E (Valencia) & 2 & Sánchez de Rivera y Moset (Madrid) & 20 \\
\hline Lozano-Monzón, R (Zaragoza) & 6 & Segarra, J (Madrid) & 34 \\
\hline Maestre, $T$ (Madrid) & 1 & Verdes Montenegro (Madrid) & 13 \\
\hline
\end{tabular}

rección del tercero de los fundadores de Murcia Médica, Antonio Guillamón-. Simultáneamente, Sánchez Pozuelos actuó como redactor responsable de «aparato Circulatorio» para otra revista murciana, Levante Médico (1928-1932).

Aunque Revista de Tisiología y Especialidades fue la primera de las sucesoras en publicarse, la heredera directa de Murcia Médica parece ser Estudios Médicos, que bajo la dirección de Sánchez Pozuelos mantuvo en su redacción a Guillamón y a Martínez Ladrón de Guevara, conservó el vínculo institucional con la Academia de Medicina de Murcia, que había establecido su antecesora, y mantuvo también la estructura de especialidades en la redacción.

\section{Murcia Médica como Órgano de la Academia de Medicina y Cirugía de Murcia}

El nombramiento como órgano oficial se produjo el 16 de abril de $1917^{42}$. Tras él se creó en la revis- ta una nueva "Sección Oficial». De esta manera la Academia de Murcia disponía de un espacio propio en el que se publicaron conferencias o discursos que hasta este momento se reunían con otros trabajos similares en la sección de "Academias y Sociedades Médicas». Esta nueva "Sección Oficial» aparece en ocho números. Era el Secretario de la Academia, con el visto bueno del Presidente, el responsable de su contenido. Se trata en realidad del primer embrión de lo que después serían los Anales de la Real Academia de Medicina y Cirugía de Murcia; de hecho, en todas las ocasiones en que aparece la sección en la revista se incluye esa denominación como segundo título, antes del artículo publicado ${ }^{43}$.

Dos días después del nombramiento de Murcia Médica como órgano oficial, en la Real Academia de Medicina y Cirugía de Murcia tuvo lugar la primera de una serie de conferencias que organizaron los jóvenes académicos correspondientes y directores de la revista ${ }^{44}$. 
Tabla 2. Relación alfabética de caricaturas en Murcia Médica

\begin{tabular}{|l|l|l|l|}
\hline CARICATURA DE & No & CARICATURA DE & No \\
\hline Albaladejo, L (Colaborador) & 44 & Medina Romero, F (Colaborador) & 42 \\
\hline Alemán, F (Redactor) & 29 & Molina Niñirola, JA (Redactor) & 28 \\
\hline Amorós, E (Redactor) & 41 & Palazón Lacárcel, E (Redactor) & 37 \\
\hline Cano Soria, F (Redactor) & 31 & Precioso Córdoba, M (Redactor) & 32 \\
\hline Egea López, J (Redactor) & 35 & Romero Elorriaga, A (Redactor) & 38 \\
\hline Gallego Alcáraz, J (Redactor) & 36 & Ruiz Medina, J (Redactor) & 30 \\
\hline Giner Hernández, F (Redactor) & 34 & Sánchez Carrascosa (Redactor) & 26 \\
\hline Guillamón Conesa, A (Director) & 24 & Sánchez Pozuelos, J (Director) & 25 \\
\hline Hernández-Ros, C (Colaborador) & 43 & Vinader Mazón, J (Redactor) & 40 \\
\hline Martínez Ladrón de Guevara, JA (Director) & 23 & & \\
\hline
\end{tabular}

\section{Estudios Médicos (1920, 1924-1934), segundo Órgano Oficial de la Real Academia de Medicina de Murcia}

Tras un par de años de haber desaparecido Murcia Médica, José Sánchez Pozuelos retomó su actividad publicista y en 1920 puso en marcha una nueva revista mensual de medicina, cirugía y especialidades, Estudios Médicos (López y Sáez, 2005; Pérez Gómez, 2011). Sánchez Pozuelos conservó como redactor jefe de Medicina a su antiguo colaborador Antonio Guillamón Conesa e incorporó como redactor jefe de Cirugía a Antonio Hernández-Ros (ver figura 5).

También contaba en la redacción con Juan Antonio Martínez Ladrón de Guevara, que simultaneaba esta tarea con la de director fundador de Revista de Tisiología y Especialidades (1919-1926) y de Levante Médico (1928-1932).

La continuidad de la nueva revista con su antecesora, su carácter general, la distribución de sus contenidos en especialidades y su continuidad como portavoz de la Academia, le dieron una notable popularidad y difusión entre los profesionales de la Medicina. Pero el comienzo no fue fácil y la publicación se interrumpió durante tres años, hasta recuperarse en 1924.

En el epígrafe de «colaboración» Estudios Médicos contó con doce médicos, todos con ejercicio en Murcia, excepto Cajal y Maestre. La "redacción» incluía 58 profesionales de diversas localidades españolas, sobre todo Madrid y Barcelona, entre los que cabe destacar a personalidades como Decref, Fidel Fernández Martínez, Fernández Sanz, Marañón, Palanca o Piga Pascual.

Estudios Médicos representó a la Academia de Medicina de Murcia desde el primer número (y a la Aca- demia de Higiene de Cataluña desde el 8 de mayo de 1925), e incluyó exclusivamente artículos científicos y discursos leídos en la propia Academia o en otras instituciones científicas. No se estructuró, por tanto, en secciones. Contó sin embargo con una publicación paralela que aparecía como Suplemento que, esta sí organizada en secciones (oficial, noticias, prensa profesional, sumarios y bibliografía) presentaba la misma información que cualquier otra revista profesional sanitaria de la época.

Estudios Médicos consiguió sobresalir en el conjunto del periodismo sanitario español, llegando a ser la revista médica de mayor tirada en España, al imprimir hasta seis mil ejemplares mensuales. No existe un motivo claro que explique la desaparición sin previo aviso de estas revistas, pero muy posiblemente no fue ajeno a ello el ambiente de inestabilidad política y social que Murcia vivía en los años 30 del pasado siglo.

\section{CONCLUSIONES}

En Murcia, como en el resto de España, el primer tercio del siglo XX fue un período de resurgir cultural y científico que se manifestó, entre otras cosas, en el interés por la edición de numerosas revistas médicas que pretendieron difundir entre los profesionales la producción científica más relevante, nacional y extranjera. En Murcia, una comunidad de economía agrícola, sin tradición científica y carente de Universidad hasta 1915 (precisamente el año en que también se funda la primera de las revistas que nos han interesado), el impulso a estas publicaciones procedió de la obra de médicos como el que nos ocupa en este artículo, José Sánchez Pozuelos. Pertenecientes por nacimiento a la burguesía murciana 
Figura 5. Primera página de Estudios Médicos 1924, 2 (21)

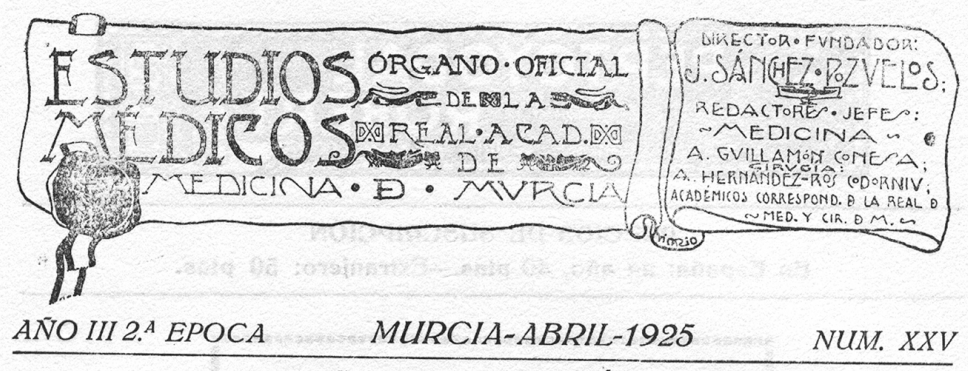

\section{COLABORACIÓN}

DOCTORES: Albaladejo, Murcia.-Ayuso, Murcia.-Cajal, Madrid.-Gomez, Murcia.-García Villalba (I.), Murcia.-Hernández-Ros, Murcia.-Maestre, Madrid.-† - Medina, Murcia.-Peña, Murcia.-Piquer, Murcia.-Quesada, Murcia.-Sánchez García, Murcia.

$$
\text { REDACCIÓN }
$$

DOCTORES: Albaladejo García, Murcia.--Albasánz, Madrid.--Alemán, Murcia.--Alvaro, Madrid.--Aroca, Murcia.--Arredondo, Madrid.--Barragán, Madrid. - Barajas, Madrid.-Bravo Moreno, Barcelona.-Blanc, Madrid.-Bové, Barcelona. - Buisan, Caspe (Zaragoza). - Calandre, Madrid. - Carrera, Palmar (Murcia).-Carro, Madrid. - Castañs, Guadalajara.-Cifuentes, Madrid.-Cilleruelo, Valladolid.-Clavero, Valladolid.-Codina, Madrid.-Decref, Madrid.Eizaguirre, San Sebastián.-Fernández Martínez, Granada.-Fernández Sánz Madrid.-Gallego, Murcia-García Triviño, Madrid.-García Hormaeche, Bilbao.-Giner, Murcia.-Goyanes, Madrid.-Hernández Ortiz, Granada.-Juarros, Madrid.-Rdo. Padre Jaime Pujiula S. J.-López Albo, Bilbao.-Lafora, Madrid.-Llorens Moltó, Málaga.-López Ambit, Murcia.-López Fanjul, Oviedo.-Mangada, Alicante.-Marañón, Madrid.-Martín, Barcelona.-Muñoz Beato, Cádiz.-Marín Agramunt, Valencia.-Martínez L. de Guevara, Murcia.-Muñoyerro, Madrid.-Martínez Serón, Murcia.-Martínez Vargas, Barcelona.-Mayoral, Madrid.-Meseguer Pardo, Murcia.-Mesonero Romanos, Madrid.-Molina Niñirola, Murcia.-t Muñoz Urra, Talavera de la Reina (Toledo).-Nubiola, Barcelona.-Novoa Santos, Santiago de Compostela (Coruña).--Palanca, Madrid.-Parache, Madrid.-Pascual Murcia, Murcia.-Pascual Rios, Madrid. -Pastor, Valencia.-Peset, Valencia.-Piga, Madrid.-Piñerúa, Madrid.-Rodríguez Arias, Barcelona.-Romero, Murcia.-Rovira, Barcelona.-Rosique, Barcelona.-Ruiz Medina, Murcia.-Salvat, Barcelona.-Sánchez Carrascosa, Murcia.-Sánchez Parra (E.) Murcia.-Sánchez Parra (R.), Murcia.-Sánchez de Rivera, Madríd.-Sánchez de Val, Cartagena (Murcia).-Sanchís Banús, Madrid.-Sáinz de Aja, Madrid.-Sánchez Covisa (I. y I.), Madrid.-Sánchez Guisande, Sevilla.-Sardina, Murcia.-Seiquer, Murcia.-Ubeda, Madrid.Vega Villalonga, Valladolid.-Villa, Madrid.-Vigueras, Madrid.

\begin{tabular}{|c|c|}
\hline Redactor Artístico: D. LU & GIL DE VICARIO. Murcia \\
\hline 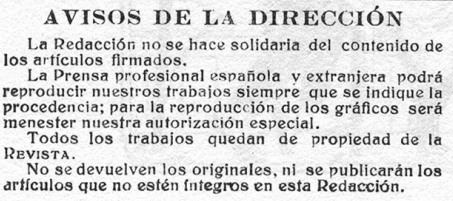 & 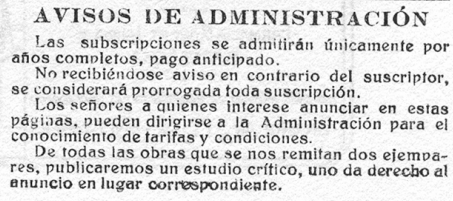 \\
\hline
\end{tabular}

que detentaba el poder político local, e identificados con ideologías de carácter conservador y religioso, al mismo tiempo se preocuparon por sacar del atraso a su tierra. A diferencia de otros que adquirieron una sólida formación científica en instituciones nacionales y extranjeras y orientaron su actividad hacia la investigación, éstos se orientaron hacia el ejercicio privado de la profesión médica y materializaron su aportación en la creación de revistas que procuraron vincular a las instituciones (en nuestro caso la Real Academia de Medicina de Murcia). De esta manera Murcia Médica (1915-1918) y Estudios Médicos (1920, 1924-34) se convirtieron en la herramienta de difusión de la actividad de esta institución mur- ciana, pero también de los profesionales murcianos y españoles, y de los trabajos más relevantes publicados en otras revistas nacionales y extranjeras; la segunda de estas revistas llegó a ser la de mayor tirada nacional. El ambiente de inestabilidad social que precedió al golpe de estado militar y el enfrentamiento bélico que le siguió truncaron el desarrollo de estas publicaciones y de los propios profesionales que las impulsaron. Ninguna de las revistas murcianas sobrevivió a la Guerra Civil, Sánchez Pozuelos fue fusilado al iniciarse la guerra y muchos de los médicos mencionados más arriba tuvieron que marchar al exilio o sufrieron un auténtico exilio interior, marginados y relegados. 


\section{NOTAS}

1 A esta fecha corresponde el testamento del médico Alonso de Espejo, en el que creó una Fundación cuya dotación se destinaba a este objetivo. Testamento de Alonso de Espejo. ES.30030. AHP/24 / Notariado de la Provincia de Murcia (1450-1913). > 24.030. / Notarías de Murcia (1450-1913). > 24.030.su / Suárez, Pedro: Protocolos, Murcia (1599-1622). > NOT,2098 / Registro de Pedro Suárez, Murcia de 1622., fols. 245-250. Archivo General de la Región de Murcia.

2 La única documentación que conserva el Archivo General de la Región de Murcia sobre Sánchez Pozuelos se refiere a su actividad religiosa. No se ha conservado su expediente en la Universidad Complutense ni en el Archivo Histórico Nacional. La referencia a la fecha de su licenciatura en Medicina procede de la prensa local que anuncia el regreso del joven médico a la ciudad y al mismo tiempo da la noticia del premio recibido por éste (diploma y medalla de oro) en el "Congreso de Sanidad de Berlín» por el específico «ARIVLE» de su propia creación. Este producto era un reconstituyente para las enfermedades del pulmón, preparado a base de glicerofosfato de cal, sulfoguayacolato de potasio y arrhenal, una sal arsenical de amplia difusión (Real Academia Nacional de Medicina, 1902). "Nuevo médico», El Liberal (Murcia), 07 diciembre 1909, p. 2. "Sánchez Pozuelos», El Tiempo (ed. mañana), 08 diciembre 1909, p. 3; El Tiempo (ed. tarde), 08 diciembre 1909, p. 2.

3 «Natalicio», El Liberal (Murcia), 05 enero 1912, p. 3

4 «Natalicios», El Tiempo (ed. mañana) 19 mayo 1917, p. 2.

5 «Patronato de San José. Una conferencia», El Tiempo (ed. tarde) 27 enero 1913, p. 2.

6 «Homenajes merecidos», La Verdad 01 enero 1920, p. 2.

7 «Los Caballeros Carmelitanos», Levante Agrario 25 agosto 1929, p. 2.

8 "Caballeros Carmelitanos», El Tiempo (ed. mañana) 26 mayo 1932, p. 2.

9 "Vida corporativa. La cofradía de la sangre», El Liberal (Murcia) 31 enero 1930, p. 4

10 «Muy llustre Cofradía de la Preciosísima Sangre», El Tiempo (ed. mañana) 31 enero 1935.

11 Juan de la Cierva Peñafiel (Mula, Murcia, 11 de marzo de 1864 - Madrid, 11 de enero de 1938), tras ser designado alcalde de Murcia en 1895 fue ministro de Instrucción Pública y Bellas Artes (1904-1905), de Gobernación (1907-1909), de Guerra (19171918, 1921-1922), y de Hacienda y de Fomento $(1919,1931)$.

12 Juan de la Cierva Codorníu (Murcia, 21 de septiembre de 1895 - Croydon, Reino Unido, 9 de diciembre de 1936), inventor del autogiro, al estallar la Guerra Civil se convirtió en eficaz colaborador de las fuerzas sublevadas.

13 "La Juventud Conservadora de Murcia», La Época (Madrid) 04 enero 1914, p. 1.

14 "La Juventud Conservadora», El Tiempo (ed. mañana) 01 enero 1915 , p. 2.
15 «Patria», El Tiempo (ed. mañana) 04 enero 1915, p. 3.

16 «Del Municipio. Una carta del Sr. Sánchez Pozuelos y un leve comentario», Levante Agrario 21 mayo 1929. "Inauguración solemne de la Casa de Teléfonos», La Verdad 19 mayo 1929, p. 1. «Inauguración de la nueva Cárcel», Flores y Naranjos 16 junio 1929, 2 (24), p. 18. "El alcalde a Madrid», Levante Agrario 26 enero 1930, p. 1. «Hablando con el alcalde», Levante Agrario 11 febrero 1930, p. 1.

17 «Noticias», Levante Médico 1929 feb; 2 (14), p. 113.

18 «De Rincón de Seca. Campaña contra la Anquilostomiasis», Levante Agrario 12 enero 1930, p. 4.

19 "En la nueva Casa de Socorro», Levante Agrario 15 diciembre 1929, p. 1. "Una visita a la Casa de Socorro», La verdad (Extraordinario) 1930 ene, pp. 4-7. «Una visita a la nueva Casa de Socorro», Flores y Naranjos 08 enero 1930, 3 (38), pp. 15-6.

20 «Ayer en el Ayuntamiento. Sesión de la Permanente», El Tiempo (Murcia) 06 febrero 1930, p. 1.

21 «Gobierno Civil. La Junta Provincial de Beneficencia», El Tiempo (Murcia) 09 febrero 1935, p. 4.

22 "Moción de la Dirección de "Estudios Médicos»», Suplemento de Estudios Médicos 1928, 6 (69), pp. 1-3. Días después la prensa local de Murcia se hizo eco de la noticia, es el caso de Levante Agrario que apoya la moción de Sánchez Pozuelos desde el 12 de diciembre de 1928.

23 José Pérez Mateos (Murcia, 2-9-1884 - 16-7-1956), médico otorrinolaringólogo, fundó en 1910 y presidió, entre 1913 y 1917, la Juventud Conservadora Maurista en Murcia. Fue alcalde de Murcia en 1921 y 1922. En 1927 fue elegido Presidente de la Federación Nacional de Colegios Médicos (puesto que ocupó hasta 1936) y desde esta posición impulsó la creación de Previsión Médica Nacional. El 22 de diciembre del 1933 a propuesta del Ministro de Gobernación, Rico Avelló, fue designado Subsecretario de Sanidad y Beneficencia, hasta octubre de 1934.

24 Mariano Ruiz-Funes García (Murcia, 24-2-1889 - México, D.F., 1953). Jurista especializado en derecho penal, fue un reconocido criminalista, premio Lombroso en Turín en 1927 por su obra Endocrinología y criminalidad. Contribuyó a la definición de figuras delictivas como las de genocidio, o el crimen de guerra. Vinculado al republicanismo de izquierdas fue uno de los diputados de la comisión que redactó el proyecto de constitución de 1931. Fue Ministro de Agricultura (1936) con Azaña, y de Justicia (1936) con Largo Caballero. (Ruiz-Funes, 2005)

25 En dicha moción Sánchez Pozuelos relató la visita de Ferrán a Murcia, en noviembre de 1926, invitado por él mismo, «a poner gratuitamente inyecciones de su vacuna "anti-alfa» a los asilados de las Casas de Misericordia e Inclusa». "Labor Municipal (continuación)», Levante Agrario 28 noviembre 1929 , p. 4. El periódico reproduce literalmente la moción de Sánchez Pozuelos. 
26 "La estancia en Murcia del autogiro Cierva», El Tiempo (Murcia) 06 septiembre 1930, p. 1. "El autogiro en Murcia», El Liberal (Murcia) 06 septiembre 1930, p. 1. «El homenaje de Murcia al inventor del autogiro señor Cierva y Codorníu», $L a$ Verdad 06 septiembre 1930, p. 1. «Con solemnidad y brillantez, el alcalde descubre la lápida que da el nombre de Cierva Codorníu a la Plaza del Rollo». El Tiempo (ed mañana) 25 abril 1935, p. 1.

27 «Nota del Tribunal Especial Popular de Murcia», El Tiempo (ed mañana) 28 noviembre 1936, p. 6. «El Tribunal Popular. Conclusiones provisionales del fiscal en la causa contra veinticuatro procesados de la capital», Nuestra Lucha 03 diciembre 1936, p. 4. «Ayer se practicó una extensa prueba de testigos de descargo», El Liberal (Murcia) 11 diciembre 1936, p. 2.

28 «Última hora. A las cuatro menos cuarto de hoy, el Tribunal ha hecho pública la sentencia condenando a muerte a trece de los procesados», Nuestra Lucha 13 diciembre 1936, p. 1. "Termina la vista de la causa contra los 24 procesados de Murcia. Se condena a pena de muerte a trece de los procesados», El Tiempo (ed. mañana) 13 diciembre 1936, p. 6.

29 «Cumplimentación de una Sentencia», Nuestra Lucha 16 diciembre 1936, p. 4.

30 «De Beniel. Viajero», El Tiempo (ed. tarde) 02 junio 1915, p. 1.

31 "Estado sanitario en Murcia», El Liberal (Murcia) 14 octubre 1918 , p. 1.

32 «Ayuntamiento de Murcia», El Tiempo (ed. mañana) 25 septiembre 1920, p. 3.

33 «La Farmacia Sánchez Pozuelos», El Tiempo (ed. mañana) 01 abril 1922, p. 1. "Una inauguración. Farmacia y Laboratorio Sánchez Pozuelos», La Verdad 01 abril 1920, p. 1.

34 «Noticias», Murcia Médica 1917, 3 (22), p. 59

35 «Nombramientos», Suplemento de Estudios Médicos 1925, 3 (27), p. 1.

36 La biografía y obra científica de los citados está siendo estudiada en la actualidad en nuestro grupo.

37 No ha sido posible localizar la publicación, que solo conocemos a través de las referencias en la prensa local: «El nuevo Reglamento de la "Asociación de Caballeros Carmelitanos"», El Tiempo (Murcia) 28 junio 1931, p. 1. "Bibliografía», La Verdad 28 junio 1931 , p. 1.

38 «Hemos cumplido el primer aniversario de nuestra publicación». Murcia Médica 1916, 2 (13), p. 231.

39 El procedimiento, afirmaron, era incluir menos publicidad pero a un precio considerablemente alto; conseguían financiar así la revista e incluso mejorar su aspecto al eliminar el exceso de publicidad, lo que además repercutía en mayores ventas. En su opinión, su estrategia no solo era inteligente, sino que también suponía un estímulo para la literatura médica española al abaratar los precios finales de suscripción. «Cómo hacemos Murcia Médica». Murcia Médica 1917, 3 (29), pp. 471-3.
40 Es curioso este hecho porque hasta en dos ocasiones se repitió la fotografía de un autor. Sucede con Emilio Bové Piqué (de Barcelona) en los números 32 y 41, y con Fermín Muñoz Urra (de Talavera de la Reina) en los 33 y 39.

41 Luis Gil de Vicario (Burgos 1898-Barcelona 1978) era un joven y recién llegado dibujante cuando comenzó a colaborar con Murcia Médica. Practicante de todos los géneros del arte (escritor, pintor, ilustrador gráfico, crítico, escultor y profesor), alcanzó un gran nivel y publicó en diarios, revistas, carteles, y otras publicaciones. Se considera que junto con Garay, Gil de Vicario protagoniza la renovación de las artes plásticas en los años veinte en Murcia y fue una figura relevante en la educación artística española. También colaboró en otras revistas médicas murcianas como Revista de Tisiología y Especialidades y Levante Médico. En los años treinta se trasladó a Barcelona donde trabajó en la Universidad como profesor de dibujo y fue comisionado en ampliación de estudios a varios países europeos.

42 La prensa murciana publicó esta noticia al día siguiente y Murcia Médica en el número de ese mismo mes, manifestando su agradecimiento "por lo mucho que vale la distinción honrosa que se nos hace, deseando que la intensa labor de la Academia con la modesta ayuda de nuestra publicidad sirva de estímulo para el estudio y contribuya a que se eleve el nivel cultural de la clase médica de nuestra región. » Murcia Médica 1917, 3 (25), p. 236.

43 Las bases primera y segunda del acuerdo por el que la revista pasaba a ser el órgano oficial entre la Academia, firmado en sesión de 16 mayo de 1917, rezan así: "1a Murcia Médica no sólo seguirá publicando los trabajos de la Real Academia, sino que establecerá una sección especial e independiente destinada a la misma, donde se inserten trabajos literarios, reseñas de sesiones, discursos doctrinales y de recepción, memorias de fin de curso, comunicaciones, concursos, premios, publicaciones recibidas, anuncios, informes oficiales, etc., en una palabra, todo cuanto la Real Academia tenga por conveniente, relacionado con ella y como si dicha sección fuera su boletín, anales o como quiera llamarse; todo lo cual será facilitado por el Académico Secretario, con el V.o B.ㅇ del Presidente. $2 \stackrel{a}{a}$ Los Directores-Propietarios de Murcia Médica conservarán su absoluta independencia y exclusiva responsabilidad en el resto de las secciones de que consta la revista;" Murcia Médica 1917, 3 (26), p. 277.

44 La conferencia fue impartida por Gómez Ocaña, profesor de Fisiología de la Facultad de Medicina de Madrid, y su tema fue «Del ejercicio y del reposo, del ayuno y del régimen alimenticio, como agentes terapéuticos». El acto finalizó con un discurso del Presidente de la Academia, Francisco Medina, en el que felicitó a los organizadores, por el éxito del primer acto, y al conferenciante, a quien entregó el título de Académico Corresponsal, por su discurso -Albaladejo, Laureano (1918), «Memoria reglamentaria de los trabajos de esta Real Academia de Medicina del año anterior (Sesión inaugural del curso de 1918)», Murcia Médica, 4 (35), pp. 85-9 -. «En la Real Academia de Medicina. Conferencia del Sr. Gómez Ocaña», El Tiempo (ed. mañana) 19 mayo 1917 , p. 1. 


\section{BIBLIOGRAFÍA}

Capel Sáez, Horacio; Joan Eugeni Sánchez y Omar Moncada (1988), De Palas a Minerva. La formación científica y la estructura institucional de los ingenieros militares en el siglo XVIII, Barcelona, Ed. del Serbal-CSIC.

Carrillo, Juan Luis (2013), “De la biografía individual a la biografía colectiva: una experiencia desde la historia de la ciencia", Erebea. Revista de Humanidades y Ciencias Sociales, (3), pp. 227-247.

Ferrándiz Araujo, Carlos (1994), “La Real Academia de Medicina de Cartagena: Aspectos institucionales y bibliométricos a través de su órgano Oficial 'La Unión de Ciencias Médicas'”, Anales de la Real Academia de Medicina y Cirugía de Murcia, pp. 111-72.

Gastón Barcos, Mariano y Mạ. Isabel Ubieto Artur (1996), Periodismo Médico Aragonés. Clínica y Laboratorio (1905-1912), Zaragoza, Anubar Ediciones.

Guillamón, Antonio (1929), “Campaña contra la Anquilostomiasis en la Huerta de Murcia. Memoria de los trabajos realizados por la Oficina Sanitaria de Puebla de Soto en el año 1928", Estudios Médicos, 6 (70), pp. 2-16, p. 16.

López González, José (2012), La labor del doctor Juan Antonio Martínez Ladrón de Guevara en el Periodismo médico murciano (1915-1932), tesis doctoral, Murcia, Facultad de Medicina, Universidad de Murcia.

López González, José y José Miguel Sáez Gómez (2005), “El periodismo profesional y científico en las instituciones sanitarias murcianas". En: Valera Candel, Manuel (ed.), Ciencia e Instituciones Científicas en la Región de Murcia (1750-1936), Murcia, Fundación Séneca. Consejería de Educación y Cultura, pp. 273-92.

López González, José; José Miguel Sáez Gómez y Manuel Valera Candel (2002), "Recuperación y análisis del periodismo profesional sanitario de la Región de Murcia en el período de entreguerras (1919-1938)". En: Fernández Sanz; Rueda Laffond; Sanz Establés (eds.), Prensa y Periodismo Especializado (historia y realidad actual). I Congreso Internacional de prensa y periodismo especializado, Guadalajara, Ayuntamiento de Guadalajara, pp. 209-26.

López Piñero, José María y María Luz Terrada (1991), Bibliografía Médica Hispánica 1475-1950. Vol. IX. Bibliometría de las revistas 1736-1950, Valencia, Instituto de Estudios Documentales e Históricos sobre la Ciencia.

Marset Campos, Pedro (2008), Médicos murcianos de la escuela histológica española, Murcia, Editum.

Marset Campos, Pedro y José Miguel Sáez Gómez (2013), “La ciencia médica en la segunda parte del franquismo. Los inicios de la Facultad de Medicina de Murcia (1968-1976)". En: Marset, Pedro; José Miguel Sáez Gómez y Carlos López Fernández (dir.) Ciencia e Instituciones Científicas en la Región de Murcia. El Franquismo, Murcia, Editum, pp. 667-720.

Moreno Fernández, Luis Miguel (1987), Acción Popular Murciana. La derecha confesional murciana durante la II República, Murcia, Universidad de Murcia.
Moreno Fernández, Luis Miguel (1996), "La C.E.D.A. y los Medios de Comunicación Social en Murcia durante la II República", Anales de Historia Contemporánea, 12, pp. 459-69.

Olagüe de Ros, Guillermo (2005), "De las vidas ejemplares a las biografías colectivas de los médicos. Una perspectiva crítica", Asclepio, 57 (1), pp. 135-148.

Pérez Gómez, Cayetano (2011), La Real Academia de Medicina y Cirugía de Murcia a través del periodismo médico murciano (1907-1933), tesis doctoral, Murcia, Facultad de Medicina, Universidad de Murcia.

Real Academia Nacional de Medicina (1902), “Dictamen de la Sección de Medicina sobre una memoria que trata de Arrhenal en el paludismo", Anales de la Real Academia de Medicina, Tomo XXII, Cuadernos 2 y 3, Madrid, Real Academia Nacional de Medicina, pp. 270-7.

Rodríguez Llopis, Miguel (1999), Historia de la Región de Murcia, Murcia, Editora Regional.

Rodríguez Ruiz, Purificación (2012), Antonio Pedro Rodríguez Pérez (1912-1964): un murciano en la Escuela Neurohistológica Española, tesis doctoral. Murcia, Universidad, Departamento de Ciencias Sociosanitarias.

Ruiz-Funes, Concepción (2005), "Mariano Ruiz Funes”, Anales de Derecho, 23, pp. 329-342.

Rumeu de Armas, Antonio (1980), Ciencia y tecnología en la España ilustrada, Madrid, Turner.

Sáez Gómez, José Miguel (1992), Sociología de los profesionales sanitarios murcianos del siglo XVIII, Ayuntamiento de Cartagena.

Sáez Gómez, José Miguel y José López González (2005), “La introducción del especialismo médico en las instituciones sanitarias murcianas". En: Valera Candel, Manuel (ed.) Ciencia e Instituciones Científicas en la Región de Murcia (1750-1936), Murcia, Fundación Séneca, pp. 273-292.

Sáez Gómez, José Miguel y Pedro Marset Campos (2013), “Academia de Medicina y Colegio de Médicos en el franquismo". En: Marset, Pedro; José Miguel Sáez Gómez y Carlos López Fernández (dir.), Ciencia e Instituciones Científicas en la Región de Murcia. El Franquismo, Murcia, Editum, pp. 511-572.

Sáez Gómez, José Miguel; Pedro Marset Campos y José López González (2008), "Luis Gil de Vicario y su contribución artística a la prensa médica murciana del primer tercio del siglo XX", Murgetana, 59 (119), pp. 141-54.

Sáez Gómez, José Miguel; Pedro Marset Campos y Cayetano Pérez Gómez (2013), “Las instituciones médicas murcianas en el primer tercio del siglo XX: Real Academia de Medicina y Colegio de Médicos". En: Marset, Pedro; José Miguel Sáez y Carlos López (dir.), Ciencia e Instituciones Científicas en la Región de Murcia. El franquismo (1936-1975), Murcia, Editum, pp. 455-510. 
Sánchez Pozuelos, José (1915a), “Arritmias”, Murcia Médica, 1 (1), pp. 17-9.

Sánchez Pozuelos, José (1915b), “Un caso de estrechez mitral curado con inyecciones de Fibrolixina”, Murcia Médica, 1 (3), pp. 104-5.

Sánchez Pozuelos, José (1916), "Algunas consideraciones sobre la fisiología del corazón", Murcia Médica, 2 (10), pp. 6-11.
Sebastián Raz, José Manuel (2011), Ciencia y compromiso: Luis Calandre Ibáñez, su vida y obra, Murcia, Editum.

Valera Candel, Manuel (2005). "La Universidad de Murcia y el inicio de la enseñanza científica superior en la Región murciana". En: Valera Candel, Manuel (ed.) Ciencia e Instituciones Científicas en la Región de Murcia (1750-1936), Murcia, Fundación Séneca, pp. 293-316. 